\title{
Morphological study of myelinated and unmyelinated fibres in the sacrococcygeal dorsal roots of the rat
}

\author{
J.-C. Lee' ${ }^{1}$, C.-H. Cheng' ${ }^{2}$, C.-T. Yen ${ }^{1}$ \\ ${ }^{1}$ Department of Life Science, National Taiwan University, Taipei, Taiwan \\ ${ }^{2}$ Department of Veterinary Medicine, National Taiwan University, Taipei, Taiwan
}

[Received: 22 August 2018; Accepted: 3 October 2018]

Background: The number and calibre of myelinated and unmyelinated fibres of the sacrococcygeal dorsal roots innervating the tail of rats were studied by means of light and electron microscopy.

Materials and methods: There were an estimated total of 12,500 myelinated and 25,500 unmyelinated dorsal root fibres innervating the tail of a rat.

Results: The results showed that from the second sacral (S2) to the fourth sacral (S4) segment, the fibre diameter spectrum of myelinated fibres within each dorsal root was bimodal with two peaks at 5 microns and 10 microns, respectively. The first sacral (S1) segment was composed of numerous smaller-size myelinated fibres, thus forming a right-skewed distribution. The coccygeal (Co) segments showed a unimodal distribution peaking at 10 microns for the first (Co1) segment and gradually shifting to 7 microns for the third (Co3) segment. Overall, there was a continuous relative increase of the larger vs. the smaller myelinated fibres from the sacral to coccygeal segments. The fibre diameter of unmyelinated fibres of all these roots was unimodal with a single peak at 0.5 microns. The ratio of unmyelinated to myelinated fibre numbers was on average 2.83 for the S1-S2 roots, 1.66 for the S3-S4 roots, and 1.24 for the coccygeal roots.

Conclusions: The comparison of the left- and right-side nerve fibres show that there was no significant difference, thus implying a symmetrical sensory innervation of the rat's tail. (Folia Morphol 2019; 78, 2: 267-273)

Key words: tail, dorsal root, axon diameter, frequency distribution

\section{INTRODUCTION}

Axonal size is the most important determinant of the nerve fibres' functional parameters, including their conduction velocity, refractory period, and sensory and motor functions [27, 28]. Close correlations between conduction velocity and nerve fibre diameter have been revealed in myelinated $[4,10,26,31]$ and unmyelinated fibres [20]. Therefore, it is important to ascertain the nerve fibre composition in a nerve or in a region. The evaluation of nerves' fibre populations is frequently performed by light microscopy with osmium- or silver-impregnated preparations. This technique is suitable to examine myelinated fibres. On the other hand, electron microscopy allows a correct evaluation of all fibre populations [17]; but, its high magnification sometimes makes it impractical to perform a total count of the nerve population of the whole nerve.

Address for correspondence: Dr. C.-T. Yen, Department of Life Science, National Taiwan University, Taipei, Taiwan 106, tel: +886-2-3366-2451, fax: +886-2-2363-6837, e-mail: ctyen@ntu.edu.tw 
Table 1. The numbers of unmyelinated (UMY) and myelinated (MY) fibres in sacrococcygeal dorsal roots of one exemplar rat

\begin{tabular}{|c|c|c|c|c|c|c|c|c|c|}
\hline \multirow[t]{2}{*}{ Roots } & \multicolumn{2}{|c|}{ UMY } & \multicolumn{2}{|c|}{ Sampling ratio } & \multicolumn{2}{|c|}{ MY } & \multicolumn{3}{|c|}{ C/A ratio } \\
\hline & $\mathbf{L}$ & $\mathbf{R}$ & $\mathbf{L}$ & $\mathbf{R}$ & $\mathbf{L}$ & $\mathbf{R}$ & $\mathbf{L}$ & $\mathbf{R}$ & Average \\
\hline S1 & 5049 & 5654 & $34.6 \%$ & $31.5 \%$ & 1795 & 1657 & 2.81 & 3.41 & 3.11 \\
\hline S2 & 1734 & 2248 & $34.9 \%$ & $45.9 \%$ & 1235 & 1287 & 1.4 & 1.75 & 1.58 \\
\hline S3 & 2028 & 2353 & $47.2 \%$ & $42.8 \%$ & 1037 & 1006 & 1.96 & 2.34 & 2.15 \\
\hline S4 & 1281 & 1739 & $27.8 \%$ & $21.8 \%$ & 838 & 840 & 1.53 & 2.07 & 1.8 \\
\hline Co1 & 832 & 1023 & $50.5 \%$ & $30.9 \%$ & 748 & 782 & 1.11 & 1.31 & 1.21 \\
\hline Co2 & 460 & 735 & $54.3 \%$ & $56.6 \%$ & 426 & 394 & 1.08 & 1.87 & 1.47 \\
\hline Co3 & 181 & 163 & $98.8 \%$ & $97.6 \%$ & 103 & 144 & 1.76 & 1.13 & 1.44 \\
\hline Sum & 11,565 & 13,915 & & & 6182 & 6110 & & & \\
\hline
\end{tabular}

$\mathrm{L}$ - left; $\mathrm{R}$ - right

The present study is an electron and light microscopic analysis of the number of dorsal root axons for all segments of the sacrococcygeal cord innervating the tail of the rat [33]. We estimated that a total of 12,500 myelinated and 25,500 unmyelinated dorsal root fibres innervate the tail of a rat. The ratio of unmyelinated fibres vs. myelinated fibres ranges from 3.4 to 1.1 in the individual roots. The frequency distribution of myelinated fibre diameter was bimodal with a continued shift from a smaller calibre (A delta fibre) to larger calibre (A beta fibre), from sacral to coccygeal segments. This will be discussed in relation to the differential functions of the segment organisation of the tail.

\section{MATERIALS AND METHODS}

\section{Sample preparation}

Thirteen male Wistar rats weighing 250-400 g were used in this study. Rats were anesthetised by intraperitoneal injections of sodium pentobarbital (100 $\mathrm{mg} / \mathrm{kg}$ ). When anaesthesia was deep, the right atrium was opened, and the animal was perfused through a needle with buffered saline followed by $500 \mathrm{~mL}$ of fixative which contained $4 \%$ paraformaldehyde and $1 \%$ glutaraldehyde in $0.1 \mathrm{M}$ phosphate buffer, $\mathrm{pH}$ 7.4. After perfusion ceased, the laminectomy was performed from segments L6 to Co3. Dorsal roots were then identified and removed from the animal. A $5 \mathrm{~mm}$-long piece of central process was cut about $2 \mathrm{~mm}$ away from the dorsal root ganglion. The roots were then rinsed with buffer and placed in fresh fixative for $2 \mathrm{~h}$ and then placed in 1\% osmium tetroxide in $0.1 \mathrm{M}$ phosphate buffer, $\mathrm{pH}$ 7.4. The tissue was again rinsed, dehydrated in ascending concentrations of ethanol then acetone, and embedded in the Spurr plastic.

\section{Histological methods}

From each specimen, we prepared semithin sections stained with toluidine blue for light microscope obser- vations. Light microscopic photographs of nerve sections were obtained at a final magnification of $400 \times$. From these photographs, we counted the number and obtained the morphometric histograms of myelinated fibres. The myelinated fibres with calibre equal or smaller than 6.5 micron were assigned as A-delta group, whereas those larger than 6.5 micron were assigned as A-beta group. The ultrathin (gold) sections were placed on formvar-coated copper grids. These sections were stained with $0.2 \%$ lead citrate and $1 \%$ uranyl acetate and photomicrographic montages of the nerve at $4000 \times$ magnification were made. Using the transmission electron microscope (JEOL: JEM-1200 EXII, USA), we photographed the nerve's total surface. The photographs were then overlapped partly in order to reconstruct the whole nerve. The montaged photography of the whole nerve was used in counting the total number of unmyelinated fibres and to identify the location of each and every Remak bundle. All Remak bundles were further photographed at $7500 \times$ for morphometric analysis. Since the copper grid blocked part of the sample, the counting of unmyelinated fibres was carried out on the observable region only. The total number of unmyelinated fibres in the whole roots was estimated using the ratio of the sampling area (Table 1).

\section{Morphometric analysis}

The contact print photographs were measured with a computer-operated planimeter (Jandel corp., USA). The fibres were traced one by one, and the calibre areas were measured and written to a worksheet. Diameters were computed by taking areal measurements and calculated assuming a perfect round shape. Therefore, the diameters are abstraction numbers.

\section{Statistical methods}

The data was expressed in terms of mean values \pm standard deviations (SD). The ratio of A-delta vs. 


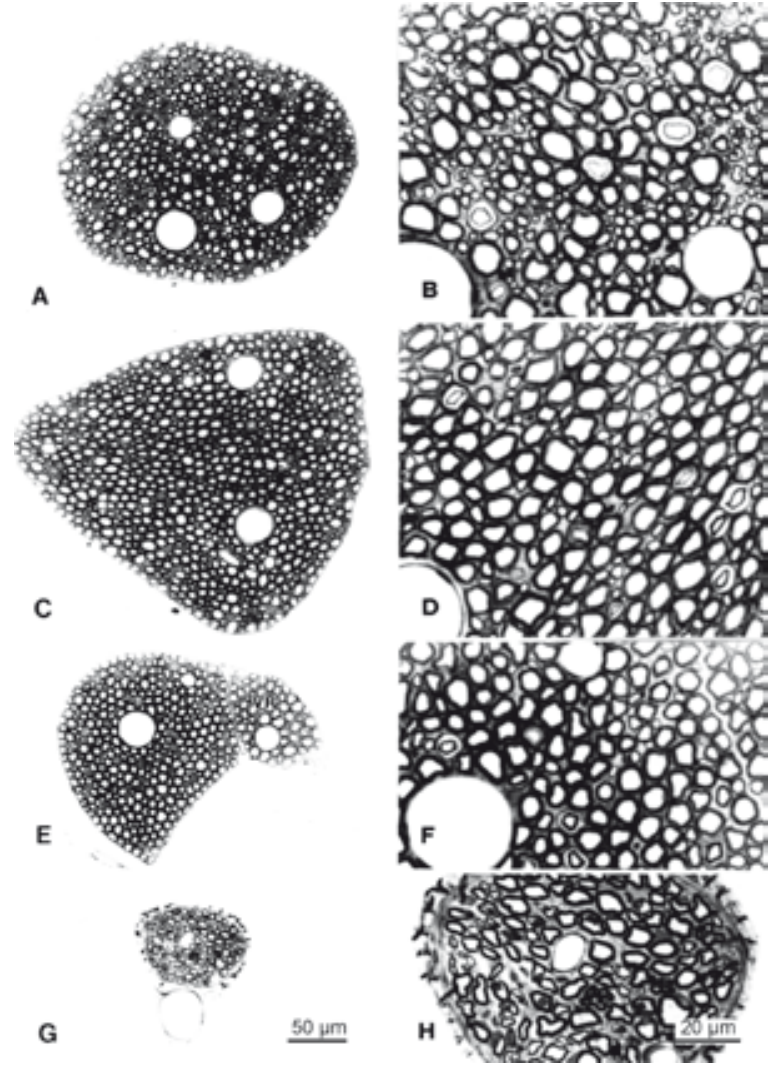

Figure 1. Representative examples of photomicrographs of myelinated fibres in several sacrococcygeal dorsal roots S1 (A, B), S3 (C, D), Co1 (E, F) and Co3 (G, H). The whole roots are shown on the left $(A, C, E, G)$, and their enlarged parts are shown on the right $(B, D, F, H)$. The myelinated axons and blood vessels are the most obvious components. The ventral root adheres to the dorsal root in the first coccygeal sample (E). S1 contains a greater proportion of smaller myelinated axons compared to the other segments.

A-beta fibre was analysed by one-way ANOVA on rank followed with post-hoc multiple comparison (Dunn's Method, Sigmaplot v.11) where appropriate. The significant level was $p<0.05$.

\section{RESULTS}

Dorsal roots consist of myelinated and unmyelinated axons embedded in a collagenous connective tissue. Perineurial cells line each root and blood vessels are prominent within them. Figure 1 illustrates the morphology of exemplar S1, S3, Co1, and $\mathrm{Co} 3$ dorsal roots in semi-thin sections. The myelinated axons and blood vessels are the most obvious components. Note that the myelinated axons in the $\mathrm{S} 1$ contain a greater proportion of smaller axons compared to the other segments. The myelinated axons can be counted with both the light and electron microscopes, because they are surrounded by an easily visible sheath. The unmyelinated axons are round or irregularly oval on cross section and are embedded

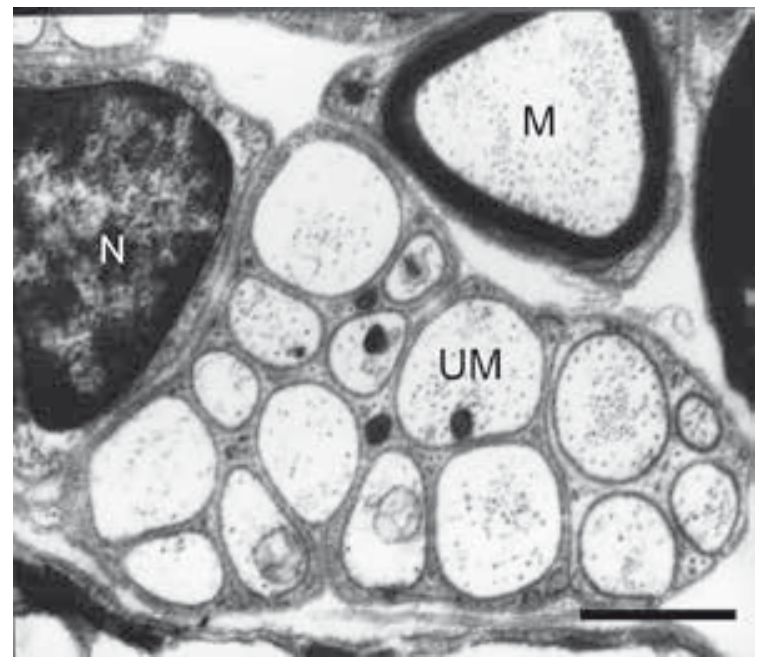

Figure 2. An electron micrograph of a typical group of unmyelinated axons (a Remak bundle) in a rat dorsal root. Note the large prominent nucleus of the Schwann cell in the left-hand side of the picture. The unmyelinated axons are smaller, irregularly round, or oval profiles embedded in troughs of Schwann cell cytoplasm. Edges of larger myelinated axons can be seen at the upper-right corner of this micrograph. $\mathrm{M}$ - myelinated axon; UM — unmyelinated axon; $\mathrm{N}$ - nucleus of the Schwann cell. Calibration bar is 1 micrometre.

in troughs of Schwann cell cytoplasm (Fig. 2). The major organelles in these axons are neurofilaments and microtubules but mitochondria and cisternae of the agranular endoplasmic reticulum can occasionally be seen.

Myelinated axon numbers were obtained by total counting, while unmyelinated axon numbers by partial sampling. The sampling ratio varied from $21.8 \%$ to $98.8 \%$ (Table 1). We estimated that a total of 12,500 myelinated and 25,500 unmyelinated dorsal root fibres innervate the tail of a rat. The ratio of unmyelinated fibres vs. myelinated fibres (C/A ratio) ranges from 3.4 to 1.1. Note the ratio in the S1 dorsal root was much larger than in other segments (Table 1). The data pooled from 11 rats are shown in Table 2 .

The fibre diameter frequency distribution of myelinated fibre (Fig. 3) was bimodal and unmyelinated fibre (Fig. 4) was unimodal. It can be seen that there are two or three peaks in the S1 dorsal roots. Note that the $\mathrm{S} 1$ roots had a high proportion of small fibres whereas those of $\mathrm{S} 2-\mathrm{S} 4$ have a greater proportion of medium and large size fibres with peaks at 5 microns and 10 microns.

The myelinated fibre histogram indicates a decline change from $\mathrm{S} 1$ to $\mathrm{Co} 2$ in the proportion of small calibre A-delta ( $\leq 6.5$ micron) vs. large calibre A-beta (> 6.5 micron) fibres. The $\mathrm{S} 1$ segment was composed of a significantly larger portion of small A-delta fibres than the S4, Co1, and Co2 segments (Fig. 5). Note 
Table 2. The numbers of unmyelinated (UMY, C fibre) and myelinated (MY, A fibre) fibres in sacrococcygeal dorsal roots of 11 rats

\begin{tabular}{|c|c|c|c|c|c|c|c|}
\hline \multirow[t]{2}{*}{ Root } & \multicolumn{3}{|c|}{ UMY } & \multicolumn{3}{|c|}{ MY } & \multirow[t]{2}{*}{$\mathrm{C} / \mathrm{A}$ ratio } \\
\hline & Mean & SD & $\mathbf{N}$ & Mean & SD & $\mathbf{N}$ & \\
\hline S1 & 4959 & 899 & 6 & 1659 & 184 & 10 & 2.99 \\
\hline S2 & 2984 & 1023 & 6 & 1147 & 106 & 7 & 2.60 \\
\hline S3 & 1828 & 484 & 6 & 931 & 142 & 9 & 1.96 \\
\hline S4 & 1305 & 472 & 4 & 642 & 234 & 4 & 2.03 \\
\hline Co1 & 813 & 165 & 4 & 568 & 176 & 11 & 1.43 \\
\hline Co2 & 577 & 121 & 4 & 504 & 230 & 9 & 1.14 \\
\hline Co3 & 172 & 13 & 2 & 189 & 101 & 7 & 0.91 \\
\hline Sum & 12,637 & & & 5640 & & & \\
\hline
\end{tabular}

SD - standard deviation

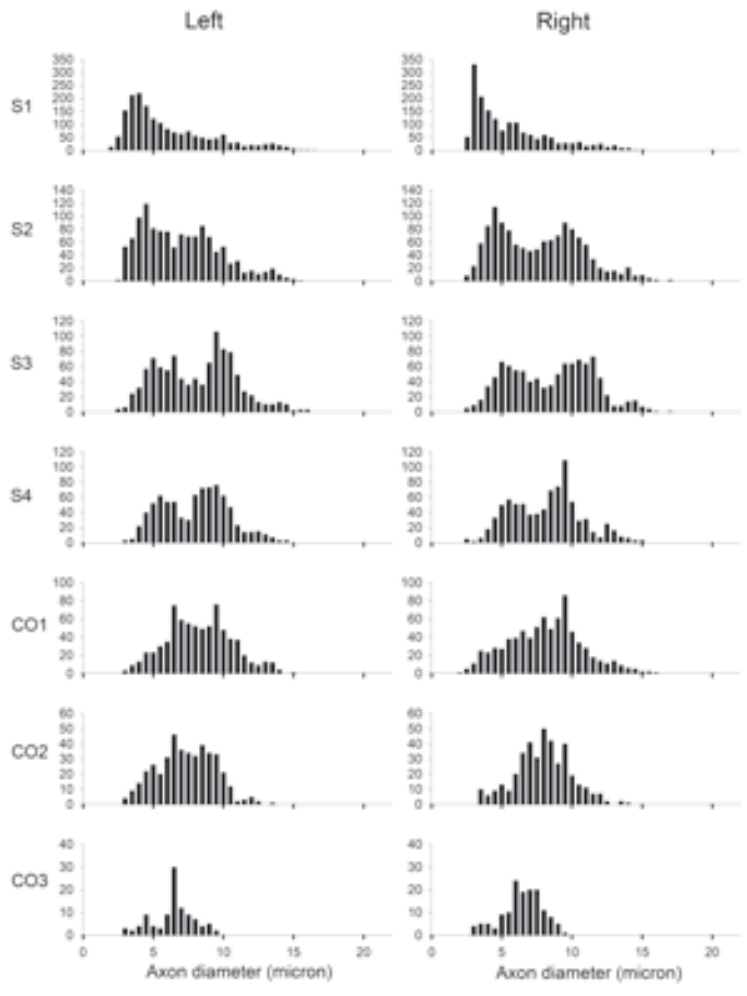

Figure 3. Examples of histograms of myelinated axon fibre size of sacrococcygeal dorsal roots in one rat. Note bimodal distribution of fibre size in the S2 to S4 dorsal roots. The A-delta fibres were most abundant in the S1 dorsal roots, whereas their peaks were much lower in the coccygeal segments.

the Co3 segment also contained a larger portion of A-delta fibres, but not reaching significant level. The fibre spectrum of unmyelinated fibres of these roots was unimodal with a single peak at 0.5 microns (Fig. 4). The fibre number ratio of unmyelinated vs. myelinated fibres (C/A ratio) was 2.83 for the $\$ 1-\$ 2$ roots, 1.66 for the S3-S4 roots, and 1.24 for the coccygeal roots, with a decreasing trend from sacral to coccygeal segments. Note the C/A ratio value of

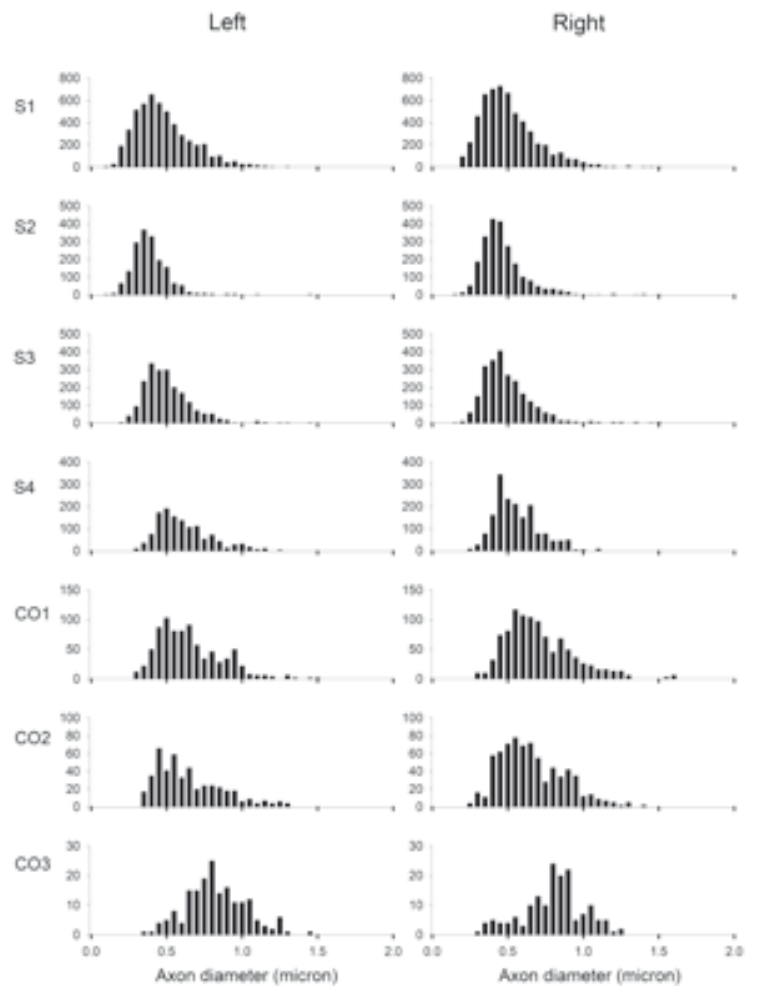

Figure 4. Examples of histograms of unmyelinated axon fibre size of sacrococcygeal dorsal roots in one rat. Note unimodal distribution of the fibre size in all segments.

S1 was much larger than other roots, although it did not reach significant level by ANOVA analysis.

\section{DISCUSSION}

The tail of the rat is a prominent organ with multiple functions. In an adult rat, the tail is over 20 $\mathrm{cm}$. It serves as the fifth limb of the rat for reaching, holding, balancing, and climbing. The tail of the rat is innervated at its base by the first segment of the sacral 


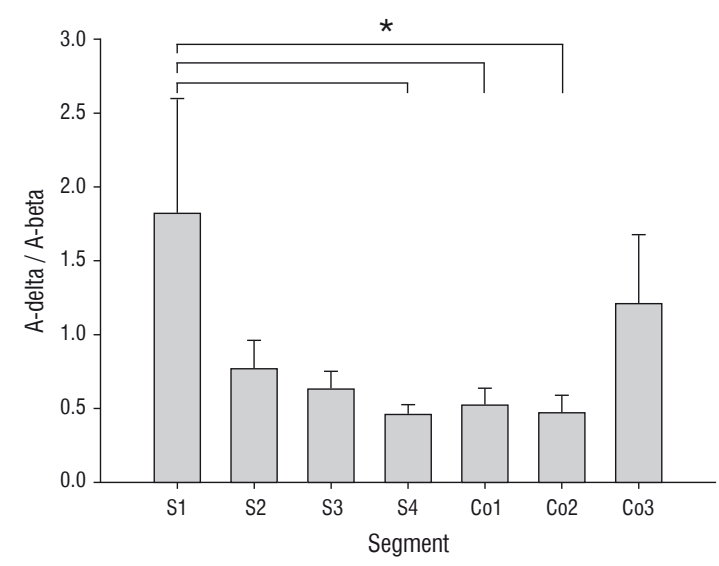

Figure 5. Ratio of A-delta vs. A-beta myelinated fibres. The S1 segment contained a significantly larger portion of $A$-delta fibres compared with $\mathrm{S} 4, \mathrm{C} 01$, and $\mathrm{Co} 2$ segments. Note the Co3 segment was also composed of a large portion of A-delta fibres, but did not show significant differences compared with other segments. One-way ANOVA on rank with Dunn's post hoc comparison; ${ }^{*} p<0.05$.

cord (S1). There is a regularly organised dermatome from $\mathrm{S} 1$ to $\mathrm{S} 4$ followed by the coccygeal segments, from rostral tail to the most caudal tip of the tail [33].

There were remarkable differences in the proportions of axons of different size categories in the roots we examined. For example, the S1 dorsal roots had a large proportion of small myelinated fibres and unmyelinated fibres. The meaning of this phenomenon is not clear, but one possible correlation is that the S1 roots in the rat are an important supplier of the sensory innervation of the pelvic viscera. This might imply that the sensory visceral innervation is predominantly by small cells. The axon-calibre histogram of dorsal roots differ on a segmental basis, which may provide clues for experiments designed to provide insights into the organisation of segmental sensory input in the spinal cord.

It is now possible to estimate precisely the total sensory and motor population of nerve fibres in the dorsal and ventral roots of the rat, including unmyelinated fibres using electron microscopy. The number of myelinated fibres established in the present study is comparable to that obtained with electron microscopy. The data of the unmyelinated component is regularly underestimated by light microscopy. This is true for certain somatic nerves [30] and also for the dorsal roots. We estimated a total of 25,500 unmyelinated and 12,500 myelinated fibres in the sacrococcygeal (S1-Co3) dorsal roots of adult Wistar rats. Compared with a previous study on counting nerve fibres of S1-S3 dorsal roots of adult Sprague-Dawley rats [18], our data showed an estimation of $21 \%$ less unmyelinated fibres and $39 \%$ less myelinated fibres in Wistar rats. The different species of the rats may contribute to the differences in nerve fibre population. However, Langford and Coggeshall's study [18] was performed 5-7 days after cutting the ventral root and sympathectomy surgery whereas our study kept all components intact. The injury of the neural tissue would trigger the development of the adjacent intact component to compensate for the loss of function and even to cause neuropathic outcomes. The sprouting of nerve branches caused by injury has been reported in DRG $[6,21]$, spinal cord [5], and dorsal roots [15]. The composition of the sciatic nerve was determined with more myelinated fibres in the sympathectomy group compared with control group [29]. We propose that the injury of ventral roots and sympathetic nerve may cause neuroplasticity and affect the morphometric analysis.

The ratio of unmyelinated to myelinated fibres is at least 1.1 for the total fibre population at the coccygeal level, but it can be greater than 3 for the sacral component. It should be emphasized that this C/A ratio is comparable to the situation observed in somatic nerves; Serratrice et al. [30] found the ratio of 4 for the sensory population of the sural nerve. At the thoracic level of the vagus nerve, this proportion is much higher since the vagi that enter the abdominal cavity consist almost entirely of $\mathrm{C}$ fibres. This has been demonstrated both by histological $[1,12,14]$ and electrophysiological studies $[16,19$, $22,25]$. On the other hand, we find at this level a clear overlapping of spectra of the unmyelinated and myelinated fibres. This shows that the classification of the smallest myelinated fibres and the largest C fibres is difficult using only diameter as a criterion. Nevertheless, the unmyelinated fibres are usually in a unimodal distribution, as in somatic nerves [23].

There is no clear consensus in the literature regarding the actual shape of nerve fibres in the transverse section. Some researchers suggest that the minimum diameter represents the true diameter. Others claim means of two orthogonal diameters. Still others accept every irregular shape as the true shape and calculate the diameter from traced circumferences or areas [13]. Orgel (1980) [24] has noted that as long as one measurement method is used consistently throughout a study, this issue can be disregarded.

A central dogma of vertebrate anatomy is that ventral spinal roots contain motor fibres, the cell bodies of which are in the spinal cord, and dorsal roots con- 
tain sensory fibres, with cell bodies in the dorsal root ganglion. However, several investigators have shown by both anatomical and physiological methods that sensory fibres are also present in the ventral roots [7]. In spinal segments $\mathrm{S} 3$ and caudal $\mathrm{Co} 1$ of the cat, which contain part of the parasympathetic outflow, $29 \%$ of the axons are unmyelinated [2]. Approximately half of these unmyelinated fibres are preganglionic efferent axons, and the rest are sensory [11]. In the rat, $30 \%$ of the axons of ventral roots L6 and S1 are unmyelinated [9]. Thirty per cent of these unmyelinated fibres arise from dorsal root ganglion cells. The other $70 \%$ arise from the spinal cord and are presumed to be preganglionic parasympathetic axons. The variable distribution also occurs in the rat [8] where ventral roots $\mathrm{T} 1-\mathrm{L} 2, \mathrm{~L} 6$, and $\mathrm{S} 1$ contain many unmyelinated and small myelinated axons, and segments $\mathrm{C} 1-\mathrm{C} 8$ and L3-L5 contain few. During this study, we observed several of the coccygeal ventral roots (e.g., Fig. 1E), and found that the unmyelinated fibre composed less than $10 \%$ of the total axon number. The number decreased from $\mathrm{Co} 1$ to $\mathrm{Co} 3$, and was much less than that of the sacral ventral roots. This observation implies that our investigation of the tail might have been slightly underestimated for around $10 \%$.

There has been extensive study of peripheral and spinal nociceptive neurons [3, 32]. Nociceptive information is carried by two populations of peripheral fibres: thinly myelinated A-delta fibres and unmyelinated $C$ fibres. A decrease in the ratio of the thinly myelinated and the unmyelinated fibres in the caudal segments of the coccygeal level, which innervate the tip of the tail, implies that the sensitivity for pain is lower in the tip of the tail. This fits well with our own observation that the laser heat sensitivity of the tailtip is the lowest compared to that of the base or the middle part of the tail.

\section{CONCLUSIONS}

A holistic picture of an organ's total innervation is important for understanding the function of that organ. In this study, we revealed the afferent organisation of the tail and demonstrated the continually changed calibre profile of nerve fibres. The description of the tail's whole sensory innervation can be a useful data point for comparison with other organs or limbs of the rat, as well as those of other species.

\section{REFERENCES}

1. Agostoni E, Chinnock JE, Daly MB, et al. Functional and histological studies of the vagus nerve and its branches to the heart, lungs and abdominal viscera in the cat. J Physiol. 1957; 135(1): 182-205, indexed in Pubmed: 13398974.

2. Applebaum ML, Clifton GL, Coggeshall RE, et al. Unmyelinated fibres in the sacral 3 and caudal 1 ventral roots of the cat. J Physiol. 1976; 256(3): 557-572, indexed in Pubmed: 1271293.

3. Besson JM, Chaouch A. Peripheral and spinal mechanisms of nociception. Physiol Rev. 1987; 67(1): 67-186, doi: 10.1152/ physrev.1987.67.1.67, indexed in Pubmed: 3543978.

4. Boyd IA, Kalu KU. Scaling factor relating conduction velocity and diameter for myelinated afferent nerve fibres in the cat hind limb. J Physiol. 1979; 289: 277-297, indexed in Pubmed: 458657.

5. Carlton S, Coggeshall R. Sprouting and reorganization in the spinal cord after nerve injury. Mechanisms and Mediators of Neuropathic Pain. 2002: 89-106, doi: 10.1007/9783-0348-8129-6_6.

6. Chung K, Lee BH, Yoon YW, et al. Sympathetic sprouting in the dorsal root ganglia of the injured peripheral nerve in a rat neuropathic pain model. J Comp Neurol. 1996; 376(2): 241-252, doi: 10.1002/(SICI)1096-9861(19961209)376:2<241::AIDCNE6>3.0.CO;2-3, indexed in Pubmed: 8951640.

7. Coggeshall RE, Coulter JD, Willis WD. Unmyelinated axons in the ventral roots of the cat lumbosacral enlargement. J Comp Neurol. 1974; 153(1): 39-58, doi: 10.1002/ cne.901530105, indexed in Pubmed: 4817345.

8. Coggeshall RE, Ito H. Sensory fibres in ventral roots L7 and Si in the cat. J Physiol. 1977; 267(1): 215-235, indexed in Pubmed: 559756.

9. Coggeshall RE. Law of separation of function of the spinal roots. Physiol Rev. 1980; 60(3): 716-755, doi: 10.1152/ physrev.1980.60.3.716, indexed in Pubmed: 6994143.

10. Cragg BG, Thomas PK. The relationships between conduction velocity and the diameter and internodal length of peripheral nerve fibres. J Physiol. 1957; 136(3): 606-614, indexed in Pubmed: 13429525.

11. Emery DG, Ito $H$, Coggeshall RE. Unmyelinated axons in thoracic ventral roots of the cat. J Comp Neurol. 1977; 172(1): 37-47, doi: 10.1002/cne.901720103, indexed in Pubmed: 838878.

12. Evans DH, Murray JG. Histological and functional studies on the fibre composition of the vagus nerve of the rabbit. J Anat. 1954; 88(3): 320-337, indexed in Pubmed: 13192020.

13. Ewart DP, Kuzon WM, Fish JS, et al. Nerve fibre morphometry: a comparison of techniques. J Neurosci Methods. 1989; 29(2): 143-150, indexed in Pubmed: 2770338.

14. Gabella G, Pease HL. Number of axons in the abdominal vagus of the rat. Brain Res. 1973; 58(2): 465-469, indexed in Pubmed: 4756138.

15. Hulsebosch CE, Coggeshall RE. Quantitation of sprouting of dorsal root axons. Science. 1981; 213(4511): 10201021, indexed in Pubmed: 7268404.

16. Iggo A. The electrophysiological identification of single nerve fibres, with particular reference to the slowest-conducting vagal afferent fibres in the cat. J Physiol. 1958; 142(1): 110-126, indexed in Pubmed: 13564422.

17. Kissin I, Freitas $C F$, Mulhern $\mathrm{HL}$, et al. Sciatic nerve block with resiniferatoxin: an electron microscopic study of unmyelinated fibers in the rat. Anesth Analg. 2007; 105(3): 825-831, doi: 10.1213/01.ane.0000277491.40055.47, indexed in Pubmed: 17717246. 
18. Langford LA, Coggeshall RE. Branching of sensory axons in the peripheral nerve of the rat. J Comp Neurol. 1981; 203(4): 745-750, doi: 10.1002/cne.902030411, indexed in Pubmed: 7328204.

19. Leek BF. Abdominal and pelvic visceral receptors. Br Med Bull. 1977; 33(2): 163-168, indexed in Pubmed: 324560.

20. Matsumoto G, Tasaki I. A study of conduction velocity in nonmyelinated nerve fibers. Biophys J. 1977; 20(1): 1-13, doi: 10.1016/S0006-3495(77)85532-X, indexed in Pubmed: 901899.

21. McLachlan EM, Jänig W, Devor $M$, et al. Peripheral nerve injury triggers noradrenergic sprouting within dorsal root ganglia. Nature. 1993; 363(6429): 543-546, doi: 10.1038/363543a0, indexed in Pubmed: 8505981.

22. Mei N. Mecanorecepteurs vagaux digestifs chez le chat. Exp Brain Res. 1970; 11(5), doi: 10.1007/bf00233971, indexed in Pubmed: 5490689.

23. Ochoa J, Mair WG. The normal sural nerve in man. I. Ultrastructure and numbers of fibres and cells. Acta Neuropathol. 1969; 13(3): 197-216, indexed in Pubmed: 5805973.

24. Orgel MGA. Critical review of histological methods used in the study of nerve regeneration. Nerve Repair and Regeneration: Its clinical and experimental basis. St Louis: Mosby. 1980: 141-148.

25. Paintal AS. Vagal Afferent Fibres. Ergebnisse der Physiologie Biologischen Chemie und Experimentellen Pharmakologie. 1963: 74-156, doi: 10.1007/978-3-642-49896-1_3.
26. Ritchie JM. On the relation between fibre diameter and conduction velocity in myelinated nerve fibres. Proc $\mathrm{R} S \mathrm{Soc}$ Lond B Biol Sci. 1982; 217(1206): 29-35, doi: 10.1098/ rspb.1982.0092, indexed in Pubmed: 6131421.

27. Rushton WAH. A theory of the effects of fibre size in medullated nerve. J Physiol. 1951; 115(1): 101-122, indexed in Pubmed: 14889433.

28. Schalow G, Zäch GA, Warzok R. Classification of human peripheral nerve fibre groups by conduction velocity and nerve fibre diameter is preserved following spinal cord lesion. J Auton Nerv Syst. 1995; 52(2-3): 125-150, indexed in Pubmed: 7615895.

29. Schmalbruch H. Fiber composition of the rat sciatic nerve. Anat Rec. 1986; 215(1): 71-81, doi: 10.1002/ ar.1092150111, indexed in Pubmed: 3706794.

30. Serratrice G, Mei N, Pelissier JF, et al. Cutaneous, muscular and visceral unmyelinated afferent fibres: comparative study. Peripheral Neuropathies. 1978: 69-82, doi: 10.1016/b978-0-444-80079-4.50010-7.

31. Waxman SG. Determinants of conduction velocity in myelinated nerve fibers. Muscle Nerve. 1980; 3(2): 141-150, doi: 10.1002/mus.880030207, indexed in Pubmed: 6245357.

32. Willis WD. The pain system. The neural basis of nociceptive transmission in the mammalian nervous system. Pain Headache. 1985; 8: 1-346, indexed in Pubmed: 2983301.

33. Yen $C T$, Chen RS. Tail region of the primary somatosensory cortex and its relation to pain function. Novel Trends in Brain Science. 2018: 233-252, doi: 10.1007/978-4-431-73242-6 14. 